This is the final peer-reviewed accepted manuscript of:

D. Belli, S. Chessa, B. Kantarci and L. Foschini, "Toward Fog-Based Mobile Crowdsensing Systems: State of the Art and Opportunities," in IEEE Communications Magazine, vol. 57, no. 12, pp. 78-83, December 2019.

The final published version is available online at: http://dx.doi.org/10.1109/MCOM.001.1900003

Rights / License:

The terms and conditions for the reuse of this version of the manuscript are specified in the publishing policy. For all terms of use and more information see the publisher's website. 


\title{
Towards Fog-Based Mobile Crowdsensing Systems: State of the Art and Opportunities
}

\author{
Dimitri Belli $^{1}$, Stefano Chessa ${ }^{1}$, Burak Kantarci ${ }^{2}$, Luca Foschini ${ }^{3}$ \\ ${ }^{1}$ Dipartimento di Informatica, University of Pisa, Italy, \{dimitri.belli, stefano.chessa\}@di.unipi.it \\ ${ }^{2}$ School of Electrical Engineering and Computer Science, University of Ottawa, Canada, burak.kantarci@uottawa.ca \\ ${ }^{3}$ Dipartimento di Informatica, Scienza e Ingegneria, University of Bologna, Italy, luca.foschini@unibo.it
}

\begin{abstract}
MCS is an emerging paradigm that leverages the pervasiveness of mobile, wearable, and vehicle-mounted devices to collect data from urban environments for ubiquitous service provisioning. In order to manage MCS application data streams efficiently, a scalable computing infrastructure hosting heterogeneous and distributed resources is critical. FC, as a geodistributed computing paradigm, is a key enabler for this requirement as it bridges cloud servers and smart mobile devices. Research on the integration of MCS with FC has recently started to be explored recognizing the requirements of MCS and their coexistence with the cyberphysical systems. In this article, we analyze the state of the art of FC solutions in MCS systems. After brief overview of MCS we emphasize the link between MCS and the FC. We then investigate the existing fog-based MCS architectures in detail by focusing on their building blocks, as well as the challenges that yet remain un-addressed. Our detailed review on the subject results in a taxonomy of FC solutions in MCS systems. In particular, we highlight the node structures, the information exchanged, the resource and service management, and the type of solutions adopted concerning privacy and security. Moreover, we provide a thorough discussion on the open issues and challenges by reporting useful insights for the researchers in MCS and FC.
\end{abstract}

\section{INTRODUCTION}

Mobile Crowd-Sensing (MCS) is an ubiquitous sensing concept that emerged with the widespread use of mobile and wearable devices. MCS leverages the computational capabilities of user device sensors, as well as their short-range communication interfaces so as to sense and share data collected inside a region of interest where users (participants) move and interact.

Despite the benefits of employing MCS to achieve sensing as a service model, various challenges remain before MCS can be widely adopted. These challenges include energy concerns due to battery limitation of devices, limited computational resources, and privacy, security, and trustworthiness of crowdsensed data. Concerning the energy consumption and computational resources, participatory or opportunistic use of built-in sensors may result in fast drain of batteries and slowing down of other applications running on the device. Security and privacy in MCS is a two-fold concern considering both the possibility of uncovering regular behavioural patterns of users through continuously collected sensory data and ensuring the veracity of crowdsensed data due to heterogeneity of participants. Moreover, in MCS, finding efficient task assignment strategies is a grand challenge, and associated to the privacy concerns, when participants are averse to share their locations. In MCS platforms, the use of intermediary nodes is not foreseen, and both storage and communications operations are delegated to mobile nodes and back-end server. When intermediary nodes are present, they are usually selected in a non-arbitrary way: those devices are selected based on their computational capacities, energy resources and memory. In any case, the cost for the entire platform remains high, especially for those devices replacing the functions of others. Some of these problems can be easily overcome by adding an intermediate layer between the direct CloudDevice communication one. Such a new layer should provide operations for storing, processing and analytics, guaranteeing at the same time data anonymization.

Fog Computing (FC), is an extension of the Cloud Computing (CC) concept introduced by Cisco. FC has established itself as a significant standard, detailing how edge nodes belonging to the middleware layer should behave to ease the work of both mobile devices and the Cloud. As intermediate layer bridging devices and cloud central servers, FC aims at handling delay-sensitive tasks in the compute resources close to the users so to reduce communication latency towards cloud platforms. Thus, FC facilitates a hierarchical compute infrastructure based on distributed execution of information analysis at the edge, whereas it delegates the global coordination and processing tasks to central cloud servers. This mechanism introduces a three-tier hierarchical structure as follows: i) client tier that consists of the users, ii) fog tier where the fog nodes are located, and iii) central cloud servers. FC, initially coined as enabler for Internet of Things (IoT) applications and services aimed at smart spaces and big data analytics, is now successfully employed in several different areas, such as cyber-physical systems, augmented reality, and shared-parking systems. Recent projects also explored open FC standards such as a context-aware structure for network virtualization, traffic engineering, and software defined networking [1].

FC serves geo-distributed applications while MCS facilitates sensing and dissemination of data within larger circles of geographic coverage such as urban settings. Thus, when the fog architecture is bridged 


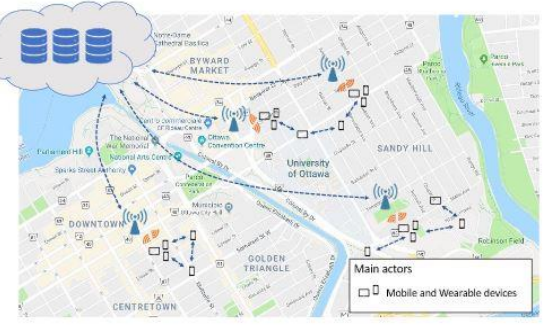

(a) A MCS System

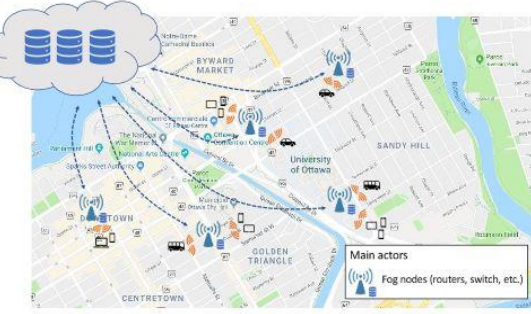

(b) A FC System

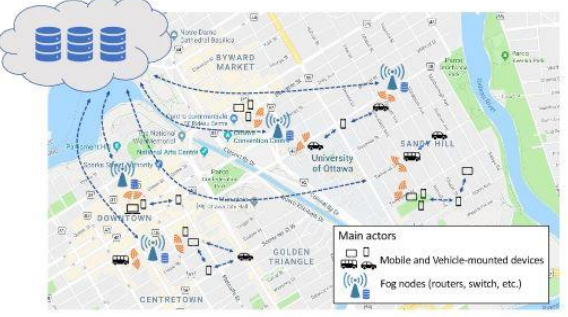

(c) A Fog-based MCS System

Fig. 1 The three considered scenarios.

with the MCS concept, low latency, bandwidth saving, and reduction of computational overhead on the devices are achieved. Furthermore, such integration ensures a scalable cost-efficient architecture and an overall improvement of the user experience. Today, the most active area that benefits from the merge of these two paradigms (i.e. FC and MCS) is vehicular sensing. [2], particularly in applications as parking navigation, road surface monitoring and traffic collision reconstruction. In these contexts, on-board sensors in vehicles are used to acquire and share data inherent phenomena of common interest, process acquired data locally, and in turn save excessive wireless bandwidth utilization for transmissions to remote cloud servers. Another interesting direction is the fog-assisted MCS frameworks for task allocation and task assignment [3].

The objective of this article is to analyse the relation between MCS and FC. The originality of this article is twofold: it defines for the first time a taxonomy of FC solutions in MCS systems; then it reviews in terms of various criteria the strategies adopted to improve both network layer and service layer in the integrated architecture. The comparison is pursued from the standpoint of the specific requirements of various MCS application integrated with FC. We must point out that our taxonomy is closely related to a recent work covering only the aspect of the FC [4]. As the MCS-fog bridging is still in its infancy, we identify the open issues, as well as challenges, and provide focused insights to address those issues in the short and medium term.

\section{MOBILE CROWDSENSING}

MCS is a sensing paradigm emerged with the convergence of participatory and opportunistic sensing. According to Guo et al., MCS is a tool to empower citizens to employ them as active participants in the data acquisition process through their mobile devices [5]. One of the most important aspects introduced by MCS is the possibility of exploiting social-technical network effects for extending the crowd by leveraging communities of volunteers.

Existing MCS platforms include two main entities:

1. Mobile devices provided with a MCS application for data collection, temporary storage, data dissemination, and the execution of local analytics.

2. Cloud servers for storage, visualization, extensive data analytics, and task assignment. MCS data plane solutions vary according to the strategy adopted for operations on remote servers.

MCS is widely used in urban settings such as monitoring of noise pollution level or traffic congestion, identification of parking availability, and monitoring the conditions of the road surface. To date, several MCS frameworks have been proposed to facilitate the MCS platforms. One above all, Vita, a renown MCS framework that supports the deployment and the management of multiple MCS applications and tasks through mobile and cloud platforms [6]. The collection of information in largescale settings and the use of centralized servers generates a series of challenges for MCS platforms such as heavier load on mobile network radio, denser traffic to cloud servers, and increased latency for information dissemination. These shortcomings can be avoided merging MCS and FC concepts in various application scenarios. For instance, in fog-based vehicular crowdsensing, fog nodes temporarily store-and-process data sensed by devices (e.g., traffic jam or the conditions of road surface) providing local services without the support of a cloud data centre [7]. [8] presents a study on the possibility of exploiting socio-technical network effects for extending the fog nodes by leveraging communities of volunteers. The work also aims at reducing installation and maintenance costs of middleware layer nodes by replacing them with mobile devices.

From an architectural standpoint, an optimal solution for remote data processing is CAROMM (Context-Aware Real-Time Open Mobile Miner), presented in [9]. CAROMM consists of two main modules for data collection and data management, and a data analysis cluster engine which acts as a bridge between the previous modules and the remote cloud manager. Another valid architectural solution for geo-social MCS platforms is McSense [10]. In this platform the entire processing workload is moved on the servers while devices are left to collect data and perform tasks. Based on the above, it is possible to classify the MCS platforms under the following two categories:

- Fully Cloud-based MCS platforms, in which the data computation is exclusively delegated to the Cloud. 
- Partially Cloud-based MCS platforms in which lightweight processing is handled at the devices before submitting to the Cloud.

In both cases the scalability of computational resources remains a grand challenge. Therefore, fog integrated MCS stands out as a promising alternative to meet scalability and efficiency objectives.

\section{FC AND MCS}

FC extends the Cloud facilities closer to devices that work with IoT data and enriches the EC middleware layer with a variety of intermediate edge entities (i.e. fog nodes) interconnected by different communication technologies. FC improves the performance of several IoT applications, including MCS, providing a structure able to analyse IoT data close to where it is collected. Energy efficiency, privacy, security, data allocation, data acquisition and data analytics are some of the aspects investigated in recent fog assisted MCS frameworks. In [3], for instance, $\mathrm{FC}$ is leveraged to assist the MCS cloud server in the assignment of tasks to the users of the platform on the basis of their mobility patterns. In this way, the platform also provides the detection of equivalent data and the prevention of duplicatelinking leakage for applications that are sensitive to the bandwidth and storage specifications. FC and MCS operate in the intermediate layer with network edge devices and end users, respectively. Amalgamation of these technologies can shift these two levels towards human-driven FC systems.

Remote servers are considered part of either fogcloud and MCS-cloud architectures. The three subfigures in Fig. 1 show an MCS system, an FC system and a possible fog-based MCS system in an urban scenario. However, there are not only benefits in the use of fog nodes in MCS platforms. For instance, incentive policies suitable only for MCS platforms needs to be rethought according the new architecture and the application scenario in which it operates, as well as users' selection strategies and techniques to guarantee a qualitative standard of the data generated. A general purpose framework to facilitate user recruitment and task completion in smart city settings is proposed in [11]. Here, fog cloudlets are deployed on bus stops of a city where devices of an MCS campaign are recruited on the basis of parameters as battery level, distance to sensing tasks, and social interaction patterns of devices' owners. Another study in [12] proposes a crowdsensing approach that recruits MCS participants cooperating through an FC-enabled IoT platform. The work relies on a business model for the fog layer involving participants of an MCS campaign, ensuring highly persistent active participation for sensing tasks. In [13], security service recommendation is considered as a use case for a social fog architecture where service provisioning depends on users' interactions. Thus, upon the discovery and the assessment of the security services, the recommender system searches for the best global security service in the service swarm by exploiting crowdsensing. The integration of MCS and FC also affects data analytics. In [14], a fogbased data-analytics scheme is proposed alongside

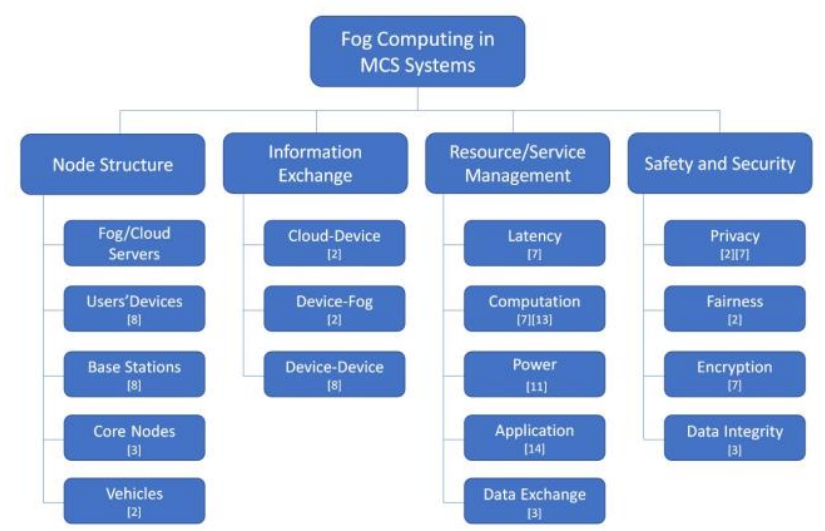

Fig. 2 Taxonomy of FC solutions in MCS systems.

cost-efficient resource provisioning for fog-based MCS in smart city MCS applications demanding real-time services. Another recent field of study that benefits from the integration of MCS and FC is the vehicular crowdsensing [2[[7]. In this context mobile devices use on-board sensors to collect and share data while fog nodes are employed to meet the demands for location-specific applications and location-aware data management such as road surface monitoring, parking navigation, and traffic collision reconstruction. Even in this context, the long-standing problem of information confidentiality persists. Of the literature so far produced, we point out the study in [15], that proposes a privacypreserving scheme for the fog layer to fulfil the anonymity and non-deniability objectives while maintaining high efficiency in terms of computation and communication costs as well.

Based on the above, we introduce a taxonomy of FC solutions in MCS systems proposing a subdivision in accordance to structural aspects of fog nodes, the organization of the information exchange between nodes, the management of resources/services provisioned by the platform, and security/safety aspects. The classification of fog entities relies on a recent taxonomy proposal [4], where the features of FC architectures are analyses from a structural, service oriented and security point of view. Concerning the classification of MCS entities, we follow the most recent literature [5]. Our taxonomy for fog-based MCS solutions is depicted in Fig. 2. The criteria in the formulation of the taxonomy is as follows:

\section{A. Node Structure}

Concerning the node structure, any device with computing, storage and network connectivity can be a potential fog node. However, only sensing, storage and network capacities are required to qualify to be an MCS node. Generally, users' devices and vehicles serve as both fog nodes and MCS nodes. Both core nodes and base stations are integral parts of the FC architectures. Core nodes are important entities of the standard fog-based infrastructure, and their function is to perform all networking activities (routing, forwarding, signal conversion, etc.). Differently, base stations are highly virtualized entities that 
TABLE I

COMPARISON OF THE FOG-MCS STUDIES IN THE NETWORK LAYER

\begin{tabular}{|c|c|c|c|c|}
\hline \multicolumn{5}{|c|}{ Network Layer } \\
\hline & $\begin{array}{c}\text { Access Network } \\
\text { Technology }\end{array}$ & $\begin{array}{c}\text { Heterog } \\
\text { eneity }\end{array}$ & Scalability & $\begin{array}{l}\text { Application } \\
\text { Protocol }\end{array}$ \\
\hline Fog-based Data Analytics for Crowdsensing [14] & NA & NA & fog scalability & NA \\
\hline FC Framework for Vehicular Crowdsensing [7] & $\begin{array}{c}\text { control center, RSU, } \\
\text { vehicle }\end{array}$ & NA & NA & $\begin{array}{l}\text { CLASC } \\
\text { scheme }\end{array}$ \\
\hline Crowdsensing Security Service Recommendation for FC [13] & $\begin{array}{l}\text { fog micro data center,fog } \\
\text { smart gateway }\end{array}$ & NA & high scalability & NA \\
\hline Localized Cooperation for Crowdsensing in FC [12] & Wi-Fi direct & NA & NA & NA \\
\hline Task Allocation and Secure Deduplication for MCS via FC [3] & RSU, gateways & NA & NA & $\begin{array}{l}\text { fog-MCS } \\
\text { task } \\
\text { assignment }\end{array}$ \\
\hline Data Acquisition in MCS through FC [11] & Wi-Fi & NA & NA & NA \\
\hline
\end{tabular}

provide data aggregation functions and personalized services at network devices, allowing seamless communication and data signal processing. From the computer sources standpoint, a cloud-based system of fog-based MCS architectures is generally centralized and made up of fog/MCS servers.

\section{B. INFORMATION EXCHANGE}

In a fog-based MCS architecture the exchange of information takes place in a bidirectional way between three different couples of entities, namely device-device, device-fog, fog-cloud. It is worth to note that a device is used to denote mobile devices or vehicular nodes. Indeed, direct communication between the Cloud and mobile devices is always possible, but this is not in scope of an investigation of FC solutions in MCS systems. In the vehicular communications context, an overview of the emergent fog-based vehicular crowdsensing paradigm is presented in [2], with an emphasis to security, privacy and fairness requirements in different application scenarios.

\section{RESOURCE AND SERVICE MANAGEMENT}

The management of resources and services play an important role for the efficiency of both fog and MCS .platforms, to improve the QoS and the user experience. Particularly, latency management is an essential requirement for both MCS and FC platforms. In this context, $\mathrm{FC}$ is tailored to serve geodistributed IoT applications, such as the MCS ones, requiring location awareness and low-latency computing. Concerning computation management, the study in [13] can be considered as a method for crowd sensing security service recommendation in FC architectures in which the computation model, as well as the parameters of security service discovery, are designed to guarantee high scalability in social FC systems. In [7] computational and storage resources are improved by RSUs that can serve as devices/roadside fog-node units with two-fold purpose: 1) Extending cloud services to the edges, and 2) bringing decisions close to the end users. Concerning the management of power resources, deploying fog-nodes near bus stops provided with power grid units is a viable solution to cope with the energy consumption of the nodes [11]. However, to the best of our knowledge there are no research efforts in the literature that propose energy saving solutions with regard to MCS units. Efficiency of data exchange between fog and MCS nodes require highly active utilization of communication resources. A careful management of such mechanisms is a crucial point for the reduction of these costs and an improvement of both QoS and QoE. To this end, in [3] a secure fog-assisted task allocation and data de-duplication scheme is proposed for communication efficiency. On the basis of local information about mobile users, the fogassisted task allocation reduces the overhead for the servers and improves accuracy and task assignment.

\section{SAFETY AND SECURITY}

The last branch of the taxonomy is inherent safety and security aspects. To date, most of the literature concerning the joint use of FC and MCS focuses on directions privacy, fairness, data encryption and data integrity aspects in vehicular networks. The study in [2] explores the typical fog-based vehicular crowdsensing architecture, highlighting several state-of-the-art security and privacy protection schemes for vehicular crowdsensing applications. As a complementary study, the authors in [15], introduce a road surface monitoring protocol that consists of devices and vehicles, RSU (fog-nodes) and cloud servers. A privacy preservation in vehicular crowdsensing-based road surface monitoring system using FC is also presented in [7]. The proposal builds on a renewed version of the certificate-less aggregate encryption scheme that targets data confidentiality, integrity, mutual authentication, anonymity and key escrow resilience. The related work reviewed under this section consists of studies that are orthogonal to several categories of the taxonomy. Tables I and II summarize and compare the characteristics related to service and network layers of the reviewed studies in this area. The service layer aspects include shortrange network access technology, heterogeneity, scalability and application protocol. The network layer aspects include battery efficiency, service provided, privacy consensus, data dimensionality and participation improvement. It is worth noting that the heterogeneity in the service layer stands for the diversity of computational fog and MCS nodes. We consider that both nodes are capable of offering infrastructure as a service for the network edge, as well as for the nodes acting as mobile sensors of an MCS campaign. The comparison between these 
TABLE II

COMPARISON OF THE FOG-MCS STUDIES IN THE SERVICE LAYER

\begin{tabular}{|c|c|c|c|c|c|}
\hline \multicolumn{6}{|c|}{ Service Layer } \\
\hline & Battery Efficiency & Type of Service & $\begin{array}{c}\text { Privacy } \\
\text { Consensus }\end{array}$ & Data Dimension & $\begin{array}{l}\text { Participation } \\
\text { Improvement }\end{array}$ \\
\hline $\begin{array}{l}\text { Fog-based Data Analytics } \\
\text { for Crowdsensing [14] }\end{array}$ & $\begin{array}{l}\text { energy-efficient strategy } \\
\text { for continuous } \\
\text { monitoring }\end{array}$ & $\begin{array}{l}\text { location,activity, } \\
\text { time, and energy } \\
\text { awarness }\end{array}$ & NA & data generators & NA \\
\hline $\begin{array}{c}\text { FC Framework for Vehicular } \\
\text { Crowdsensing [7] }\end{array}$ & NA & security services & $\begin{array}{l}\text { high security } \\
\text { level }\end{array}$ & $\begin{array}{l}\text { smart devices } \\
\text { sensors and } \\
\text { vehicles }\end{array}$ & NA \\
\hline $\begin{array}{l}\text { Crowdsensing Security } \\
\text { Service Recommendation for } \\
\text { FC [13] }\end{array}$ & NA & security services & & NA & NA \\
\hline $\begin{array}{l}\text { Localized Cooperation for } \\
\text { Crowdsensing in FC [12] } \\
\end{array}$ & NA & $\begin{array}{c}\text { remove replicated } \\
\text { data }\end{array}$ & NA & NA & \begin{tabular}{|l} 
local cooperation \\
/competition
\end{tabular} \\
\hline $\begin{array}{c}\text { Task Allocation and Secure } \\
\text { Deduplication for MCS via } \\
\text { FC [3] }\end{array}$ & NA & $\begin{array}{c}\text { remove replicate } \\
\text { data in sensing } \\
\text { reports }\end{array}$ & $\begin{array}{l}\text { security for } \\
\text { duplicate } \\
\text { attacks }\end{array}$ & NA & NA \\
\hline $\begin{array}{c}\text { Data Acquisition in MCS } \\
\text { through FC [11] }\end{array}$ & $\begin{array}{c}\text { cloudlets for energy- } \\
\text { efficient selection } \\
\text { strategy }\end{array}$ & \begin{tabular}{|c|} 
data acquisition, \\
user recruitment, \\
and task completion
\end{tabular} & NA & $\begin{array}{c}\text { accelerometer, } \\
\text { temperature, and } \\
\text { pressure }\end{array}$ & $\begin{array}{c}\text { recruitment } \\
\text { improved of } 20 \%\end{array}$ \\
\hline
\end{tabular}

approaches also focuses the Not Available aspects (NA) in both network layer and service layer. Especially the lack of details as regards heterogeneity and the application protocol highlights that the synergistic use of these technologies is still in an embryonic phase.

\section{OPEN ISSUES AND CHALLENGES}

Adaptation of FC solutions to MCS systems is a relatively recent field of study. Therefore, state of the art in this subject introduces various opportunities. First and foremost, FC inherits almost all the open issues that are still under investigation in CC, particularly those related to high vulnerability to security attacks. However, some of these problems may be transformed as a result of the combination with MCS. Below we point out these issues, challenges and opportunities on the basis of the aspects presented in the taxonomy.

\section{A. NODE STRUCTURE}

Architectural aspects can be faced in terms of optimization of power resources and communication between entities (e.g. providing frameworks for latency and overhead reduction). In the area of vehicular networks most of the studies carried out so far concerned only privacy and security issues on data transmission, but almost anything has been done about energy efficiency. With regard to services, the joint use of MCS and FC can overcome the challenges that are experienced by large-scale applications, especially those concerning the restricted resources of devices. In addition, the heterogeneity of nodes affects the communication possibilities. Particularly, communications between fog nodes are in some cases problematic due to their diversification. This aspect is currently under consideration by the scientific community because a standardization proposal has not been finalized yet.

\section{B. INFORMATION EXCHANGE}

Despite the existing behavioural studies in mobile EC and MCS domains [8], persistency of devices in specific locations of an integrated MCS-fog platform remains an open issue. As starting point to find efficient solutions on this particular aspect, the study of users' mobility and sociality is still an open research direction and a viable alternative for sharing high-quality data. As stated for node structure, heterogeneity of entities affects their communication possibilities and, consequently, makes the information exchange between fog nodes and MCS entities difficult in some cases. For instance, when fog nodes have no defined policies for associating cloud-based services spontaneously, they neglect efficient communication with remote servers and focus only on communication with devices, by restricting the potential of the entire platform. In this respect, a detailed investigation is still lacking.

\section{RESOURCE AND SERVICE MANAGEMENT}

Besides the traditional resource and service management track, social studies aiming at identifying data aggregation points can be invaluable in the identification of hot spots where to deploy base stations. Another very important aspect that has been tackled by very few researchers is the optimization of energy management methods. Moreover, the task migration to support nodes with restricted computational capacities and limited energy.power is yet to be explored. Such an aspect can bring great benefits in terms of latency reduction and energy consumption. Finally, the management of the time needed for data computation and data transmission, as well as the development of context-dependent tasks, are still open issues that await in-depth investigation. 


\section{SAFETY AND SECURITY}

Currently, research on the security of fog-based MCS platforms and solutions is limited. However, based on the review we have presented earlier, one can note that the state of the art is calling for a holistic implementation of multi-tenancy support in fog resources and scheduling according to the QoS requirements of the MCS-fog platforms. Similarly, reliability in terms of availability of highperformance services, consistency and secure fault tolerance is difficult to achieve. There exist some attempts to improve safety aspects related to communication between nodes mainly in the context of vehicular networks. Particularly, the increasing interest in Fog Vehicular Crowd Sensing (FVCS) application has opened up interesting prospects for the development of sustainable and fair incentives techniques in the field of security and privacy [2]. Data encryption plays a pivotal role in FVCS security, and the delegable key management is one of the most significant aspect to focus on. To obtain high-quality results in terms of privacy, instead, this field of study is still waiting for computationally efficient signature types that do not burden the current difficult management of pseudonyms by users and the Cloud. Ultimately, both security attacks and the implementation of security mechanisms for data-centric integrity remain open issues.

\section{E. SCALABILITY CHALLENGES}

Scalability is a fundamental aspect for MCS that can be overcome through the fog-based platforms. However, to the best of our knowledge, remarkable efforts in this field are limited to the study in [14]. The participation improvement in MCS campaigns with the support of fog architecture has only been taken into consideration by few research efforts although it is a vital aspect for such platforms [11] [12]. Moreover, a potential solution to the scalability of MCS platforms based on fog is given by studying social aspects related to the human behaviour [8]. In this context, the creation of social relations between MCS nodes in a fully independent way increases the scalability opportunities of fog platforms; this is because the interaction of mobile nodes with each other creates more possibilities to communicate sensing data to fog nodes. Finally, the lack of a fogbased MCS simulator is a brake for the possibility of testing communications between nodes in real-world or synthetic scenarios.

\section{CONCLUDING REMARKS}

The joint use of FC and MCS inherits both the benefits of MCS, such as the possibility of leveraging social-technical network effects for extending the crowd through MCS volunteers, and the advantages of FC, such as location awareness, geo-distribution, and communication efficiency.

The integration between FC and MCS would be highly beneficial in a scenario where third-party actors are able interact with private network service provider companies. The actors, for instance, may belong to small or large organizations as coffee shops or department store. Based on their purposes, such organizations might offer services on the basis of a specific business models, (e.g. support and service contracts, all-in-one enterprise cloud, etc.) to be associated to a well-defined cost model (e.g., consumption-based, advertising-based, etc.).

As fog-based MCS is an evolving and emerging area of research, we have presented a taxonomy on the basis of the most relevant goals to be achieved in this area. Then, we compared the service and network considering existing works, and we finally presented open issues, challenges and opportunities, highlighting critical aspects as the scalability of the systems, the improvement of participation, security, reliability, and energy efficiency as possible directions for future research on human-driven FC systems.

\section{REFERENCES}

[1] P. Hu, S. Dhelim, H. Ning, and T. Qiu, "Survey on fog computing: architecture, key technologies, applications and open issues," IEEE Journal of Network and computer applications, Nov 2017.

[2] . Ni, A. Zhang, X. Lin, and X. S. Shen, "Security, privacy and fairness infog-based vehicular networks," IEEE Communication Magazine, vol. 55/6, pp. 146-152, 2017.

[3] J. Ni, K. Zhang, Y. Yu, X. Lin, and X. S. Shen, "Providing task allocation and secure deduplication for mobile crowdsensing via fog computing," IEEE Transactions on Dependable and Secure Computing, 2018.

[4] R. Mahmud, R. Kotagiri, and R. Buyya, "Fog computing: a taxonomy, survey and future directions," Springer Singapore Internet of Everything. Internet of Things (Technology, Communications and Computing), pp.103130, Oct 2017.

[5] B. Guo, Z. Yu, X. Zhou, and D. Zhang, "From participatory sensing to mobile crowdsensing," IEEE Pervasive Computing and Communica-tions, pp. 593598, Mar 2014

[6] X. Hu, T. Chu, H. Chan, and V. Leung, "Vita: A crowdsensing-oriented mobile cyber-physical system," IEEE Transactions on Emerging Topics in Computing, pp. 148-165, Jul 2013.

[7] S. Basudan, X. Lin, and K. Sankaranarayanan, "A privacy-preserving vehicular crowdsensing-based road surface condition monitoring system using fog computing," IEEE Internet of Things Journal, pp. 772782, Feb 2017.

[8] S. Chessa, D. Belli, L. Foschini, and M. Girolami, "A social-based approach to mobile edge computing," IEEE Symposium on Computers and Communications, Jul 2018 .

[9] W. Sherchan, P. P. Jayaraman, S. Krishnaswamy, A. Zaslavsky, S. Loke, and A. Sinha, "Using on-the-move mining for mobile crowdsensing," IEEE MDM - Mobile Data Management, pp. 115-124, Nov 2012.

[10] G. Cardone, L. Foschini, P. Bellavista, A. Corradi, C. Borcea, M. Ta-lasila, and R. Curtmola, "Fostering participaction in smart cities: a geo-social crowdsensing platform," IEEE Communication Magazine, pp.112-119, Jun 2013

[11] C. Fiandrino, F. Anjomshoa, B. Kantarci, D. Kliazovich, P. Bouvry, andJ. N. Matthews, "Sociability-driven framework for data acquisition in mobile crowdsensing over fog computing platforms for smart cities," IEEE Transactions on Sustainable Computing, pp. 345-358, Oct 2017. 
[12] L. Jaimes, A. Chakeri, and R. Steele, "Localized cooperation for crowdsensing in a fog computing-enabled internet-of-things," Journal of Ambient Intelligence and Humanized Computing, May 2018.

[13] J. Wu, Z. Su, and J. Li, "Crowd sensing-enabling security service recommendation for social fog computing systems," Sensors, vol. 17, Jul 2017.

[14] H. R. Arkian, A. Diyanat, and A. Pourkhalili, "Mist: Fogbased data analytics scheme with cost-efficient resource provisioning for iot crowd-sensing applications," Journal of Network and Computer Applications,pp. 152-165, 2017.

[15] J. Wei, X. Wang, N. Li, G. Yang, and Y. Mu, "A privacypreservingfog computing framework for vehicular crowdsensing networks," IEEE Access, pp. 43 776-43 784, Jul 2018.

\section{AUTHORS}

Dimitri Belli received his M.S. from the University of Pisa in 2016. Currently, he is $\mathrm{PhD}$ student in Computer Science at the University of Pisa. His research interests are in mobile distributed systems, Internet of Things and smart environment with a special focus on mobile crowdsensing and multi-access edge computing paradigms.

Stefano Chessa is Associate Professor at the Department of Computer Science of the University of Pisa. He co-authored more than 150 papers published on international journals and conference proceedings. His research interests are in the areas of internet of things, mobile networks, smart environments, ambient assisted living and activity recognition.

Burak Kantarci is an Associate Professor at the School of Electrical Engineering and Computer Science of the University of Ottawa. He has co-authored over 150 refereed papers in established journals and conferences. His current research interests are IoT, vehicular networks, big data, mobile crowdsensing, social networks, and continuous authentication via behavioural biometrics. He is a Distinguished Speaker of the ACM.

Luca Foschini graduated from the University of Bologna, from which he received a Ph.D. degree in Computer Engineering in 2007. He is now an Assistant Professor of Computer Engineering at the University of Bologna. His interests include distributed systems and solutions for system and service management, management of cloud computing, context data distribution platforms for smart city scenarios, context-aware session control, multi-access edge computing, and mobile crowdsensing and crowdsourcing. 